\title{
COMPARISON BETWEEN THE LAPLACIAN-ENERGY-LIKE INVARIANT AND THE KIRCHHOFF INDEX*
}

\author{
S. PIRZADA ${ }^{\dagger}$, HILAL A. GANIE ${ }^{\dagger}$, AND IVAN GUTMAN ${ }^{\ddagger}$
}

\begin{abstract}
For a simple connected graph $G$ of order $n$, having Laplacian eigenvalues $\mu_{1}, \mu_{2}, \ldots$, $\mu_{n-1}, \mu_{n}=0$, the Laplacian-energy-like invariant $(L E L)$ and the Kirchhoff index $(K f)$ are defined as $L E L(G)=\sum_{i=1}^{n-1} \sqrt{\mu_{i}}$ and $K f(G)=n \sum_{i=1}^{n-1} \frac{1}{\mu_{i}}$, respectively. In this paper, $L E L$ and $K f$ are compared, and sufficient conditions for the inequality $K f(G)<L E L(G)$ are established.
\end{abstract}

Key words. Laplacian spectrum, Laplacian-energy-like invariant, Kirchhoff index.

AMS subject classifications. 05C50, 15A18.

1. Introduction. Let $G$ be finite, undirected and simple graph with $n$ vertices and $m$ edges having vertex set $V(G)=\left\{v_{1}, v_{2}, \ldots, v_{n}\right\}$. The adjacency matrix $A=$ $\left(a_{i j}\right)$ of $G$ is the $(0,1)$-square matrix of order $n$ whose $(i, j)$-entry is equal to one if $v_{i}$ is adjacent to $v_{j}$, and is equal to zero otherwise. Let $D(G)=\operatorname{diag}\left(d_{1}, d_{2}, \ldots, d_{n}\right)$ be the diagonal matrix, where $d_{i}$ is the degree of the vertex $v_{i}$ of $G$. Then $L(G)=D(G)-$ $A(G)$ is the Laplacian matrix, and its spectrum $S p_{L}(G)=\left\{\mu_{1}, \mu_{2}, \ldots, \mu_{n-1}, \mu_{n}\right\}$ is the Laplacian spectrum of the graph $G$. For the sake of simplicity, we use $\mu_{i}^{t_{j}}$ to denote that the eigenvalue $\mu_{i}$ is repeated $t_{j}$ times in the spectrum. In what follows, we assume that $\mu_{1} \geq \mu_{2} \geq \cdots \geq \mu_{n}$. For other undefined notations and terminology from spectral graph theory, the readers are referred to [6, 32 .

It is well known that the Laplacian eigenvalues are non-negative real numbers and that eigenvalue zero has multiplicity equal to the number of connected components of the underlying graph $G$, for more details on Laplacian eigenvalues, see 16, 18, 19, 29, 30, 33, 34, 36, 37. Thus, $\mu_{n}=0$ for all graphs, and $\mu_{n-1}>0$ if and only if $G$ is connected. The eigenvalue $\mu_{n-1}$ is called the algebraic connectivity of the graph $G$ [10, 12].

The concept of resistance distance was introduced by Klein and Randić 23]. In a graph $G$, the resistance distance between vertices $v_{i}$ and $v_{j}$, denoted by $r_{i j}$, is defined

\footnotetext{
${ }^{*}$ Received by the editors on March 31, 2015. Accepted for publication on December 31, 2015. Handling Editor: Bryan L. Shader.

${ }^{\dagger}$ Department of Mathematics, University of Kashmir, Srinagar, India (pirzadasd@kashmiruniversity.ac.in, hilahmad1119kt@gmail.com).

${ }^{\ddagger}$ Faculty of Science, University of Kragujevac, Kragujevac, Serbia, and State University of Novi Pazar, Novi Pazar, Serbia (gutman@kg.ac.rs).
} 
to be the effective resistance between nodes $v_{i}$ and $v_{j}$ as computed by Kirchhoff's laws, when all the edges of $G$ are considered to be unit resistors.

The traditional distance between vertices $v_{i}$ and $v_{j}$, denoted by $d_{i j}$, is the length of a shortest path connecting them. The Wiener index $W(G)$ is defined as $W(G)=$ $\sum_{i<j} d_{i j}$. As an analogue to the Wiener index, the sum $K f(G)=\sum_{i<j} r_{i j}$ was considered [23], later named the Kirchhoff index [5]. In [23], it was shown that $r_{i j} \leq d_{i j}$ and $K f(G) \leq W(G)$ with equality if and only if $G$ is a tree.

The Kirchhoff index has a nice purely mathematical interpretation. Mohar and one of the present authors 21] demonstrated that the Kirchhoff index of a connected graph satisfies the relation

$$
K f=K f(G)=n \sum_{i=1}^{n-1} \frac{1}{\mu_{i}}
$$

Noteworthy applications in chemistry of the Kirchhoff index as a molecular structure descriptor have been found [5, 7, 11, 31, 43. For details on the extensive mathematical research of the Kirchhoff index, see the recent papers [2, 3, 4, 14, 15, 24, 35, 38, 40, 42, and the references cited therein.

Another Laplacian-spectrum-based graph invariant was put forward by Liu and Liu [27], defined as

$$
L E L=L E L(G)=\sum_{i=1}^{n-1} \sqrt{\mu_{i}}
$$

and named this as Laplacian-energy-like invariant. The motivation for introduction of the $L E L$ was in its analogy to the earlier studied graph energy [25] and Laplacian energy [22, 25]; for more details see the survey [26] and the references cited therein. Recently, several mathematical investigations of the $L E L$ were communicated [8, 20, 28, 35, 38, 39, 41, 44, 45].

Motivated by the papers [3, 9] in which two sufficient conditions were established for the inequality $K f(G)>L E L(G)$, and the relations between $K f(G)$ and $L E L(G)$ were completely solved for trees, unicyclic graphs, bicyclic graphs, tricyclic graphs, and tetracyclic graphs, we now obtain sufficient conditions under which $L E L(G)>$ $K f(G)$ holds. Complete comparisons are only given for these graphs if they have a sufficient number of vertices. 
2. Main results. In order to compare the Kirchhoff index and the Laplacianenergy-like invariant of a graph $G$, we need the following lemmas [12, 17, 18.

LEMma 2.1. Let $G$ be a connected graph of order $n$ and let $\Delta$ be its maximum degree. Then $\Delta+1 \leq \mu_{1} \leq n$. Equality holds on the left if $\Delta=n-1$ and on the right if and only if $G$ is the join of two graphs.

LemMa 2.2. Let $G ¥ K_{n}$ be a connected graph of order $n$ and let $\delta$ be its smallest vertex degree. Then $\mu_{n-1} \leq \delta$.

LEMMA 2.3. If $0=\mu_{n}<\mu_{n-1} \leq \mu_{n-2} \leq \cdots \leq \mu_{1}$ are the Laplacian eigenvalues of the graph $G$, then the Laplacian eigenvalues of its complement $\bar{G}$ are $0=\mu_{n}<$ $n-\mu_{1} \leq n-\mu_{2} \leq \cdots \leq n-\mu_{n-1}$.

Our first result is as follows.

THEOREM 2.4. Let $G$ be a connected graph with algebraic connectivity $\mu_{n-1} \geq k$ and let $m$ be the number of edges and $\Delta$ the maximum degree of $G$. If

$$
2 m>\frac{k(\sqrt{n}+\sqrt{k})}{k+\sqrt{n}+\sqrt{k}}\left(\frac{(n+k)(n-1)}{k}-\frac{(n-1) \sqrt{k(\Delta+1)}}{\sqrt{n}+\sqrt{k}}\right),
$$

then $K f(G)<L E L(G)$.

Proof. Let $0=\mu_{n}<\mu_{n-1} \leq \cdots \leq \mu_{1}$ be the Laplacian eigenvalues of the connected graph $G$, and let $\mu_{n-1} \geq k$. Then

$$
\begin{aligned}
L E L(G) & =\sum_{i=1}^{n-1} \sqrt{\mu_{i}}=\sum_{i=1}^{n-1}\left(\sqrt{\mu_{i}}-\sqrt{\mu_{n-1}}\right)+(n-1) \sqrt{\mu_{n-1}} \\
& =\sum_{i=1}^{n-1}\left(\frac{\mu_{i}-\mu_{n-1}}{\sqrt{\mu_{i}}+\sqrt{\mu_{n-1}}}\right)+(n-1) \sqrt{\mu_{n-1}} \\
& \geq \sum_{i=1}^{n-1}\left(\frac{\mu_{i}-\mu_{n-1}}{\sqrt{\mu_{1}}+\sqrt{\mu_{n-1}}}\right)+(n-1) \sqrt{\mu_{n-1}} \\
& =\frac{2 m+(n-1) \sqrt{\mu_{1} \mu_{n-1}}}{\sqrt{\mu_{1}}+\sqrt{\mu_{n-1}}} \\
& \geq \frac{2 m+(n-1) \sqrt{(\Delta+1) \mu_{n-1}}}{\sqrt{n}+\sqrt{\mu_{n-1}}} .
\end{aligned}
$$

For $k \leq x \leq \delta$, consider the function

$$
f(x)=\frac{2 m+(n-1) \sqrt{(\Delta+1) x}}{\sqrt{n}+\sqrt{x}}
$$


for which

$$
f^{\prime}(x)=\frac{(n-1) \sqrt{n(\Delta+1)}-2 m}{2 \sqrt{x}(\sqrt{n}+\sqrt{x})^{2}} .
$$

Since $\Delta+1 \geq \frac{2 m}{n}+1 \geq \frac{2 m}{n-1}$ and $n-1 \geq \frac{2 m}{n}$, it follows that

$$
(\Delta+1)(n-1) \geq \frac{2 m}{n-1} \frac{2 m}{n}=\frac{1}{n}\left(\frac{4 m^{2}}{n-1}\right) ;
$$

that is, $(n-1) \sqrt{n(\Delta+1)}-2 m \geq 0$, implying $f^{\prime}(x) \geq 0$. Thus, $f(x)$ is an increasing function for $k \leq x \leq \delta$. Therefore, $f(x) \geq f(k)$, giving

$$
\frac{2 m+(n-1) \sqrt{(\Delta+1) x}}{\sqrt{n}+\sqrt{x}} \geq \frac{2 m+(n-1) \sqrt{(\Delta+1) k}}{\sqrt{n}+\sqrt{k}}
$$

that is,

$$
L E L(G) \geq \frac{2 m+(n-1) \sqrt{k(\Delta+1)}}{\sqrt{n}+\sqrt{k}} .
$$

We also have

$$
\begin{aligned}
K f(G) & =n \sum_{i=1}^{n-1} \frac{1}{\mu_{i}}=n \sum_{i=1}^{n-1}\left(\frac{1}{\mu_{i}}-\frac{1}{\mu_{1}}\right)+\frac{n(n-1)}{\mu_{1}} \\
& =n \sum_{i=1}^{n-1}\left(\frac{\mu_{1}-\mu_{i}}{\mu_{1} \mu_{i}}\right)+\frac{n(n-1)}{\mu_{1}} \\
& \leq n \sum_{i=1}^{n-1}\left(\frac{\mu_{1}-\mu_{i}}{\mu_{1} \mu_{n-1}}\right)+\frac{n(n-1)}{\mu_{1}} \\
& =\frac{n(n-1) \mu_{1}-2 m n}{\mu_{1} \mu_{n-1}}+\frac{n(n-1)}{\mu_{1}} \\
& \leq \frac{k n(n-1)-2 m n}{k \mu_{1}}+\frac{n(n-1)}{k} .
\end{aligned}
$$

For $\Delta+1 \leq x \leq n$, consider the function $g(x)=\frac{k n(n-1)-2 m n}{k x}$, for which $g^{\prime}(x)=$ $\frac{2 m n-k n(n-1)}{k x^{2}}>0$. As $G$ is connected, so $2 m>k(n-1)$. Therefore, $g(x)$ is an increasing function of $x$, implying $g(x) \leq g(n)$, that is,

$$
\frac{k n(n-1)-2 m n}{k x} \leq \frac{k(n-1)-2 m}{k} ;
$$

resulting in

$$
K f(G) \leq \frac{(n+k)(n-1)-2 m}{k}
$$


Suppose inequality (2.1) holds. By direct calculation, it can be transformed into

$$
\frac{2 m+(n-1) \sqrt{k(\Delta+1)}}{\sqrt{n}+\sqrt{k}}>\frac{(n+k)(n-1)-2 m}{k} .
$$

Keeping in mind (2.2) and (2.3), it follows that $L E L(G)>K f(G)$.

In particular, if $\mu_{n-1} \geq 1$, we have the result stated in Corollary 2.5. In [9, the question was raised whether it is "possible to find a constant $c$ (which may depend on the number of vertices $n$ and maximum vertex degree $\Delta$ ), such that for any connected graph $G$ with $m \geq c$ edges, $L E L(G)>K f(G)$ holds". Corollary 2.5 provides a partial answer to this question.

COROLlary 2.5. Let $G$ be a connected graph $G$ with algebraic connectivity $\mu_{n-1} \geq 1$. Let $m$ be the number of edges and $\Delta$ the maximum degree of $G$. If

$$
2 m>\frac{\sqrt{n}+1}{\sqrt{n}+2}\left(n^{2}-1-\frac{(n-1) \sqrt{\Delta+1}}{\sqrt{n}+1}\right),
$$

then $K f(G)<L E L(G)$.

Corollary 2.6. Let $T$ be a tree and $\bar{T}$ its complement. If the order of $T$ is $n \geq 7$ and $\Delta(T) \leq n-2$, then $\operatorname{LEL}(\bar{T})>K f(\bar{T})$.

Proof. Since any $T$ of order $n$ has minimum degree one and $n-1$ edges, it follows that $\Delta(\bar{T})=n-2$ and $2 m(\bar{T})=(n-1)(n-2)$. Since $\Delta(T) \leq n-2$, we have $\mu_{1}(T) \leq n-2\left(\right.$ as $\left.T \neq K_{n-1,1}\right)$ and so by Lemma $2.3, \mu_{n-1}(\bar{T})=n-\mu_{1}(T) \geq 2$. Therefore,

$$
\begin{aligned}
& \frac{2(\sqrt{n}+\sqrt{2})}{\sqrt{n}+2+\sqrt{2}}\left(\frac{(n+2)(n-1)}{2}-\frac{(n-1) \sqrt{2(n-1)}}{\sqrt{n}+\sqrt{2}}\right) \\
& =(n-1)\left(\frac{(n+2)(\sqrt{n}+\sqrt{2})-2 \sqrt{2(n-1)}}{\sqrt{n}+2+\sqrt{2}}\right) \\
& <(n-1)(n-2)=2 m(\bar{T})
\end{aligned}
$$

if

$$
n-2>\frac{(n+2)(\sqrt{n}+\sqrt{2})-2 \sqrt{2(n-1)}}{\sqrt{n}+2+\sqrt{2}},
$$

that is, $n+\sqrt{2(n-1)}>2 \sqrt{n}+4.8284$, which is true for $n \geq 7$.

Therefore, by Theorem 2.4, $L E L(G)>K f(G)$, for $n \geq 7$.

Corollary 2.7. Let $U$ be a unicyclic graph and $\bar{U}$ its complement. If the order of $U$ is $n \geq 14$ and $\Delta(U) \leq n-2$, then $L E L(\bar{U})>K f(\bar{U})$. 
Proof. Since in a unicyclic graph it is either $\delta(U)=1$ or $\delta(U)=2$, and

$$
\Delta(\bar{U})+1= \begin{cases}n-1 & \text { if } \delta(U)=1 \\ n-2 & \text { if } \delta(U)=2\end{cases}
$$

In addition, $2 m(\bar{U})=n(n-3)$ and $\mu_{n-1}(\bar{U})=n-\mu_{1}(U) \geq 1$, as $\mu_{1}(U) \leq n-1$.

If $\Delta+1=n-1$, then

$$
\begin{aligned}
\frac{\sqrt{n}+1}{\sqrt{n}+2}\left(n^{2}-1-\frac{(n-1) \sqrt{\Delta+1}}{\sqrt{n}+1}\right) & =(n-1)\left(\frac{(n+1)(\sqrt{n}+1)-\sqrt{n-1}}{\sqrt{n}+2}\right) \\
& <n(n-3)=2 m(\bar{U}),
\end{aligned}
$$

provided $n-3>\left(\frac{(n+1)(\sqrt{n}+1)-\sqrt{n-1}}{\sqrt{n}+2}\right)$, that is, $n+\sqrt{n-1}>4 \sqrt{n}+7$, which is true for $n \geq 21$.

For $n=14,15,16,17,18,19,20$, it can be checked by direct calculation that

$$
\frac{\sqrt{n}+1}{\sqrt{n}+2}\left(n^{2}-1-\frac{(n-1) \sqrt{\Delta+1}}{\sqrt{n}+1}\right)<n(n-3) .
$$

Similarly, if $\Delta+1=n-2$, then for $n \geq 14$,

$$
\frac{\sqrt{n}+1}{\sqrt{n}+2}\left(n^{2}-1-\frac{(n-1) \sqrt{n-2}}{\sqrt{n}+1}\right)<n(n-3) .
$$

Therefore, by Corollary 2.5 it follows that $L E L(\bar{U})>K f(\bar{U})$.

Corollary 2.8. Let $B$ be a bicyclic graph and $\bar{B}$ be its complement. If the order of $B$ is $n \geq 15$ and $\Delta(B) \leq n-2$, then $\operatorname{LEL}(\bar{B})>K f(\bar{B})$.

Proof. Since in a bicyclic graph, either $\delta(B)=1$ or $\delta(B)=2$, it follows that

$$
\Delta(\bar{B})+1= \begin{cases}n-1 & \text { if } \delta(B)=1 \\ n-2 & \text { if } \delta(B)=2 .\end{cases}
$$

In addition, $2 m(\bar{B})=n(n-3)-2=n^{2}-3 n-2$ and $\mu_{n-1}(\bar{B})=n-\mu_{1}(B) \geq 1$, as $\mu_{1}(B) \leq n-1$. Consider the function

$$
f(n)=n^{2}-3 n-2-\frac{\left(n^{2}-1\right)(\sqrt{n}+1)-(n-1) \sqrt{n-2}}{\sqrt{n}+2}
$$

for which

$$
f^{\prime}(n)=2 n-3-\frac{1}{(\sqrt{n}+2)^{2}}\left(2 n^{2}+\frac{13}{2} n^{3 / 2}-\frac{1}{2 \sqrt{n}}-\frac{3 n-5}{\sqrt{n-2}}-\frac{n^{2}-n-1}{\sqrt{n(n-2)}}-1\right) .
$$


It can be seen that $f^{\prime}(n)>0$, for all $n \geq 4$, that is $f(n)$ is an increasing function on $[4, \infty)$. So we have $f(n)>f(15)=0.73565404>0$, that is, $f(n)>0$ for $n \geq 15$, which implies that

$$
n^{2}-3 n-2>\left(\frac{\left(n^{2}-1\right)(\sqrt{n}+1)-(n-1) \sqrt{n-2}}{\sqrt{n}+2}\right) .
$$

The result follows from Corollary 2.5

Corollary 2.9. Let TC be a tricyclic graph and $\overline{T C}$ be its complement. If the order of $T C$ is $n \geq 16$ and $\Delta(T C) \leq n-2$, then $L E L(\overline{T C})>K f(\overline{T C})$.

Proof. Since in a tricyclic graph, either $\delta(T C)=1$ or $\delta(T C)=2$ or $\delta(T C)=3$, it follows that

$$
\Delta(\overline{T C})+1= \begin{cases}n-1 & \text { if } \delta(T C)=1 \\ n-2 & \text { if } \delta(T C)=2 \\ n-3 & \text { if } \delta(T C)=3 .\end{cases}
$$

In addition, $2 m(\overline{T C})=n(n-3)-4=n^{2}-3 n-4$ and $\mu_{n-1}(\overline{T C})=n-\mu_{1}(T C) \geq 1$, as $\mu_{1}(T C) \leq n-1$. Proceeding in an analogous manner as in the proof of Corollary 2.8, it can be shown that for $n \geq 16$,

$$
n^{2}-3 n-4>\frac{\left(n^{2}-1\right)(\sqrt{n}+1)-(n-1) \sqrt{n-3}}{\sqrt{n}+2} .
$$

The result follows from Corollary 2.5.

In a fully analogous manner, we also obtain the following.

Corollary 2.10. Let $Q C$ be a tetracyclic graph and $\overline{Q C}$ be its complement. If the order of $Q C$ is $n \geq 17$ and $\Delta(Q C) \leq n-2$, then $L E L(\overline{Q C})>K f(\overline{Q C})$.

The line graph of the graph $G$ is denoted by $£(G)$. We need the following result by Anderson and Morley [1]:

Lemma 2.11. Let $0=\mu_{n}<\mu_{n-1} \leq \mu_{n-2} \leq \cdots \leq \mu_{1}$ be the Laplacian eigenvalues of the graph $G$ and let $t_{1} \geq t_{2} \geq \cdots \geq t_{n}$ be the degree sequence of its line graph $£(G)$. Then $\mu_{1} \leq t_{1}+2$, with equality if and only if $G$ is a regular or a semiregular bipartite graph.

THEOREM 2.12. If $G$ is a graph for which $\mu_{1}<n-n^{2 / 3}$, then $L E L(\bar{G})>$ $K f(\bar{G})$.

Proof. By applying Lemma 2.3, we have

$$
\operatorname{LEL}(\bar{G})-K f(\bar{G})=\sum_{i=1}^{n-1} \sqrt{n-\mu_{i}}-\sum_{i=1}^{n-1} \frac{n}{n-\mu_{i}}=\sum_{i=1}^{n-1} \frac{\left(n-\mu_{i}\right)^{3 / 2}-n}{n-\mu_{i}} .
$$



which

For $\mu_{n-1} \leq x \leq \mu_{1}$, consider the function $f(x)=\left[(n-x)^{3 / 2}-n\right] /(n-x)$, for

$$
f^{\prime}(x)=-\frac{\left[\frac{1}{2}(n-x)^{3 / 2}+n\right]}{(n-x)^{2}}<0,
$$

for all $\mu_{n-1} \leq x \leq \mu_{1}$. Thus, $f(x)$ is decreasing for $\mu_{n-1} \leq x \leq \mu_{1}$, implying

$$
f(x) \geq f\left(\mu_{1}\right)=\frac{\left(n-\mu_{1}\right)^{3 / 2}-n}{n-\mu_{1}},
$$

that is,

$$
L E L(\bar{G})-K f(\bar{G}) \geq \frac{(n-1)\left(\left(n-\mu_{1}\right)^{3 / 2}-n\right)}{n-\mu_{1}}>0
$$

if $\left(n-\mu_{1}\right)^{3 / 2}-n>0$, i.e., $\mu_{1}<n-n^{2 / 3}$. $\square$

REMARK 2.13. By Lemma 2.11 $\mu_{1} \leq t_{1}+2$, where $t_{1}$ is the maximum vertex degree of the line graph $£(G)$. From Theorem 2.12 it follows that $f(x) \geq f\left(t_{1}+2\right)=$ $\frac{\left(n-t_{1}-2\right)^{3 / 2}-n}{n-t_{1}-2}$, which gives $L E L(\bar{G})>K f(\bar{G})$ if $t_{1}<n-n^{2 / 3}-2$.

The kite $K i_{n, \omega}$ is the graph of order $n$, obtained by attaching a pendent path on $n-\omega$ vertices to a vertex of the complete graph of order $\omega$. Let $\Gamma_{n, k}$ be the class of graphs of order $n$ obtained by attaching a pendent path on $n-k$ vertices to a vertex of a connected graph of order $k$. In particular, $K i_{n, k} \in \Gamma_{n, k}$. The following result can be found in [18, 19].

LEMma 2.14. Let $G^{\prime}=G+e$ be the graph obtained from $G$ by inserting into it a new edge e. Then the Laplacian eigenvalues of $G$ interlace the Laplacian eigenvalues of $G^{\prime}$, that is,

$$
\mu_{1}\left(G^{\prime}\right) \geq \mu_{1}(G) \leq \mu_{2}\left(G^{\prime}\right) \geq \mu_{2}(G) \geq \cdots \geq \mu_{n}\left(G^{\prime}\right) \geq \mu_{n}(G)=0
$$

Corollary 2.15. Let $G \in \Gamma_{n, k}$ with $k \geq 4$ and $n-k \geq n^{2 / 3}+2$. Then $\operatorname{LEL}(\bar{G})>K f(\bar{G})$.

Proof. Since $G \in \Gamma_{n, k}, G$ is an edge-deleted subgraph of $K i_{n, k}$. By Lemma 2.14. for $j=1,2, \ldots, n$, we have $\mu_{j}(G) \leq \mu_{j}\left(K i_{n, k}\right)$. There exists an edge $e$ in $E\left(K i_{n, k}\right)$ such that $K i_{n, k}-e=K_{k} \cup P_{n-k}$. Since $k \geq 4$, by Lemma 2.14, and in view of $\sum_{i=1}^{n}\left(\mu_{i}(G+e)-\mu_{i}(G)\right)=2$, it follows that $k+1 \leq \mu_{1}\left(K i_{n, k}\right) \leq k+2$. So 
$\mu_{1}(G) \leq \mu_{1}\left(K i_{n, k}\right) \leq k+2$. If $\mu_{1}(G)<n-n^{2 / 3}$, then $k+2<n-n^{2 / 3}$, that is $n-k>n^{2 / 3}+2$. ㅁ

Corollary 2.16. Let $G ¥ K_{n}$ be an $r$-regular graph with $n$ vertices. If $r<(n-$ $\left.n^{2 / 3}\right) / 2$, then $L E L(\bar{G})>K f(\bar{G})$. If $r>\left(n+n^{2 / 3}-2\right) / 2$, then $L E L(G)>K f(G)$.

Proof. Since $G$ is $r$-regular, its line graph $£(G)$ is $t_{1}=(2 r-2)$-regular. Assume that $r<\left(n-n^{2 / 3}\right) / 2$. Then $t_{1}=2 r-2<n-n^{2 / 3}-2$. By Remark 2.13, $L E L(\bar{G})>$ $K f(\bar{G})$.

Since $\bar{G}$ is $(n-1-r)$-regular, its line graph $£(\bar{G})$ is $t_{1}=(2 n-2 r-4)$-regular. Assume that $r>\left(n+n^{2 / 3}-2\right) / 2$. Then $t_{1}=(2 n-2 r-4)<n-n^{2 / 3}-2$. By Remark 2.13, $\operatorname{LEL}(G)>K f(G)$.

The following result has been proven in [3].

LEMma 2.17. Let $G+e$ be the graph obtained by adding a new edge to the connected graph $G$. If $K f(G)<L E L(G)$, then $K f(G+e)<L E L(G+e)$.

Let $K K_{n}^{j}$ be the graph obtained from two copies of complete graphs $K_{n}$, by joining a vertex of one copy with $j, 1 \leq j \leq n$, vertices of the other copy. The Laplacian spectrum of $K K_{n}^{j}$ was obtained in [13] and is given by

$$
S p_{L}\left(K K_{n}^{j}\right)=\left\{n^{2 n-j-2},(n+1)^{j-1}, \frac{(n+j+1) \pm \sqrt{(n+j+1)^{2}-8 j}}{2}, 0\right\} .
$$

Therefore,

$$
\begin{aligned}
L E L\left(K K_{n}^{j}\right)= & (2 n-j-2) \sqrt{n}+(j-1) \sqrt{n+1} \\
& +\sqrt{\frac{(n+j+1)+\sqrt{(n+j+1)^{2}-8 j}}{2}} \\
& +\sqrt{\frac{(n+j+1)-\sqrt{(n+j+1)^{2}-8 j}}{2}}
\end{aligned}
$$

and

$$
\begin{aligned}
K f\left(K K_{n}^{j}\right)= & \frac{2 n(2 n-j-2)}{n}+\frac{2 n(j-1)}{n+1}+\frac{2 n}{\frac{(n+j+1)+\sqrt{(n+j+1)^{2}-8 j}}{2}} \\
& +\frac{\frac{2 n}{(n+j+1)+\sqrt{(n+j+1)^{2}-8 j}}}{2} \\
= & 4 n-2 j-4+\frac{2 n(j-1)}{n+1}+\frac{n(n+j+1)}{j} .
\end{aligned}
$$


For $j \geq \frac{n}{4}$ and $n \geq 22$, it is easy to see that

$$
(2 n-j-2) \sqrt{n}+(j-1) \sqrt{n+1}>4 n-2 j-3+\frac{n(n+j+1)}{j} .
$$

Therefore, from (2.4) and (2.5), we have $L E L\left(K K_{n}^{j}\right)>K f\left(K K_{n}^{j}\right)$. Thus, using Lemma 2.17, we arrive at the following result.

TheOREM 2.18. For $j \geq n / 4$, let $K K_{n}^{j}$ be a spanning subgraph of the graph $G$. Then for $n \geq 22$ we have $\operatorname{LEL}(G)>K f(G)$.

From Theorem 2.18 we observe the following. If $G$ is a graph of order $n,(n \equiv$ $0(\bmod 8))$ having two cliques of order $n / 2$ each, such that there are at least $n / 8$ edges between a vertex in one of the cliques and $n / 8$ vertices of the other clique, then for $n \geq 44, L E L(G)>K f(G)$.

It is clear from above that $\mu_{1}\left(K K_{n}^{j}\right)=n+1<2 n-(2 n)^{2 / 3}$, for $n \geq 7$. For the complement of the graph $K K_{n}^{j}$, from Theorem 2.12, it follows that $L E L\left(\overline{K K_{n}^{j}}\right)>$ $K f\left(\overline{K K_{n}^{j}}\right)$ holds for all $n \geq 7$ and $1 \leq j \leq n-1$. Thus, using Lemma 2.17, we arrive at the following result.

THEOREM 2.19. If $\overline{K K_{n}^{j}}$ is a spanning subgraph of a graph $G$ with $2 n$ vertices, then $L E L(G)>K f(G)$ for $n \geq 7$.

The join (complete product) $G_{1} \vee G_{2}$ of the graphs $G_{1}$ and $G_{2}$ is the graph with vertex set $V\left(G_{1}\right) \cup V\left(G_{2}\right)$ and edge set consisting of all the edges of $G_{1}$ and $G_{2}$ together with the edges joining each vertex of $G_{1}$ with every vertex of $G_{2}$. The Laplacian spectrum of the join is given by the the following result [18, 19].

Lemma 2.20. If $G_{1}\left(n_{1}, m_{1}\right)$ and $G_{2}\left(n_{2}, m_{2}\right)$ are two graphs having Laplacian spectra $S_{L}\left(G_{1}\right)=\left\{\mu_{1}, \mu_{2}, \ldots, \mu_{n_{1}-1}, \mu_{n_{1}}=0\right\}$ and $\operatorname{Sp}_{L}\left(G_{2}\right)=\left\{\sigma_{1}, \sigma_{2}, \ldots, \sigma_{n_{2}-1}\right.$, $\left.\sigma_{n_{2}}=0\right\}$, then $S p_{L}\left(G_{1} \vee G_{2}\right)=\left\{n_{1}+n_{2}, n_{1}+\sigma_{1}, n_{1}+\sigma_{2}, \ldots, n_{1}+\sigma_{n_{2}-1}, n_{2}+\mu_{1}, n_{2}+\right.$ $\left.\mu_{2}, \ldots, n_{2}+\mu_{n_{1}-1}, 0\right\}$.

THEOREM 2.21. For $p \geq 4$, let $K_{p} \vee \overline{K_{r}}, 1 \leq r \leq p$, be a spanning subgraph of a graph $G$ of order $n=p+r$. Then $\operatorname{LEL}(G)>K f(G)$.

Proof. The Laplacian spectra of $K_{p}$ and $\overline{K_{r}}$ are $\left\{p^{p-1}, 0\right\}$ and $\left\{0^{r}\right\}$, respectively. Therefore, by Lemma 2.20 $S p_{L}\left(K_{p} \vee \overline{K_{r}}\right)=\left\{(p+r)^{p}, p^{r-1}, 0\right\}$. This implies

$$
\begin{aligned}
K f\left(K_{p} \vee \overline{K_{r}}\right) & =\frac{n p}{p+r}+\frac{n(r-1)}{p} \\
& \leq(p+r-1)+(p-1) \leq 2(p+r-2)
\end{aligned}
$$

and 


$$
\begin{aligned}
\operatorname{LEL}\left(K_{p} \vee \overline{K_{r}}\right) & =p \sqrt{p+r}+(r-1) \sqrt{p} \\
& \geq(p+r-1) \sqrt{p} \geq 2(p+r-2)
\end{aligned}
$$

resulting in $L E L\left(K_{p} \vee \overline{K_{r}}\right) \geq K f\left(K_{p} \vee \overline{K_{r}}\right)$. Theorem 2.21 now follows from Lemma 2.17 $\mathrm{u}$

The Laplacian spectrum of the complete bipartite graph $K_{\frac{n}{2}}, \frac{n}{2}$ is $\left\{n,\left(\frac{n}{2}\right)^{n-2}, 0\right\}$. For $n \geq 5$, this yields $K f\left(K_{\frac{n}{2}}, \frac{n}{2}\right)=2 n-3<\sqrt{n}+(n-2) \sqrt{\frac{n}{2}}=L E L\left(K_{\frac{n}{2}}, \frac{n}{2}\right)$. Then Lemma 2.17 leads to the following result.

THEOREM 2.22. If $K_{\frac{n}{2}, \frac{n}{2}}$ is a spanning subgraph of a graph $G$ of order $n$, then $K f(G)<L E L(G)$, for all $n \geq 5$.

The sufficient condition given by Theorem 2.4. seems to be useful for graphs with large number of edges and large number of vertices. We now state an analogous condition pertaining to the graph complement.

THEOREM 2.23. Let $G$ be a connected graph with $n$ vertices with largest Laplacian eigenvalue $\mu_{1} \leq \frac{n}{2}$ and algebraic connectivity $\mu_{n-1} \geq k$. If

$$
2 m<\frac{(\Delta+1)(n-\Delta-1)(n(n-1)+(n-1) \sqrt{k(n-k)})}{n(\sqrt{n-k}+\sqrt{k})+(\Delta+1)(n-\Delta-1)}
$$

then $L E L(\bar{G})>K f(\bar{G})$.

Proof. Using Lemma 2.3. since $\Delta+1 \leq \mu_{1} \leq \frac{n}{2}$, we have

$$
\begin{aligned}
K f(\bar{G}) & =n \sum_{i=1}^{n-1} \frac{1}{n-\mu_{i}}=n \sum_{i=1}^{n-1}\left(\frac{1}{n-\mu_{i}}-\frac{1}{\mu_{1}}\right)+\frac{n(n-1)}{\mu_{1}} \\
& =n \sum_{i=1}^{n-1}\left(\frac{\mu_{1}+\mu_{i}-n}{\mu_{1}\left(n-\mu_{i}\right)}\right)+\frac{n(n-1)}{\mu_{1}} \\
& \leq n \sum_{i=1}^{n-1}\left(\frac{\mu_{1}+\mu_{i}-n}{\mu_{1}\left(n-\mu_{1}\right)}\right)+\frac{n(n-1)}{\mu_{1}} \\
& =\frac{2 m n}{\mu_{1}\left(n-\mu_{1}\right)} \leq \frac{2 m n}{(\Delta+1)(n-\Delta-1)}
\end{aligned}
$$

and

$$
\begin{aligned}
\operatorname{LEL}(\bar{G}) & =\sum_{i=1}^{n-1} \sqrt{n-\mu_{i}}=\sum_{i=1}^{n-1}\left(\sqrt{n-\mu_{i}}-\sqrt{\mu_{n-1}}\right)+(n-1) \sqrt{\mu_{n-1}} \\
& =\sum_{i=1}^{n-1}\left(\frac{n-\mu_{i}-\mu_{n-1}}{\sqrt{n-\mu_{i}}+\sqrt{\mu_{n-1}}}\right)+(n-1) \sqrt{\mu_{n-1}}
\end{aligned}
$$




$$
\begin{aligned}
& \geq \sum_{i=1}^{n-1}\left(\frac{n-\mu_{i}-\mu_{n-1}}{\sqrt{n-\mu_{n-1}}+\sqrt{\mu_{n-1}}}\right)+(n-1) \sqrt{\mu_{n-1}} \\
& =\frac{n(n-1)-2 m+(n-1) \sqrt{\mu_{n-1}\left(n-\mu_{n-1}\right)}}{\sqrt{n-\mu_{n-1}}+\sqrt{\mu_{n-1}}} .
\end{aligned}
$$

For $k \leq x \leq \delta$, consider the function

$$
f(x)=\frac{n(n-1)-2 m+(n-1) \sqrt{n x-x^{2}}}{\sqrt{n-x}+\sqrt{x}}
$$

for which

$$
\begin{aligned}
f^{\prime}(x)= & \frac{1}{(\sqrt{n-x}+\sqrt{x})^{2}}\left[(\sqrt{n-x}+\sqrt{x}) \frac{(n-1)(n-2 x)}{2 \sqrt{n x-x^{2}}}\right. \\
& \left.-\left(n(n-1)-2 m+(n-1) \sqrt{n x-x^{2}}\right)\left(\frac{1}{2 \sqrt{x}}-\frac{1}{2 \sqrt{n-x}}\right)\right]>0
\end{aligned}
$$

for all $k \leq x \leq \delta$. Therefore, the function $f(x)$ is increasing for $k \leq x \leq \delta$. Therefore,

$$
f(x) \geq f(k)=\frac{n(n-1)-2 m+(n-1) \sqrt{n k-k^{2}}}{\sqrt{n-k}+\sqrt{k}}
$$

from which it follows that

$$
L E L(\bar{G}) \geq \frac{n(n-1)-2 m+(n-1) \sqrt{n k-k^{2}}}{\sqrt{n-k}+\sqrt{k}} .
$$

Keeping in mind the relations (2.7) and (2.8), inequality (2.6) can be transformed into

$$
\frac{2 m n}{(\Delta+1)(n-\Delta-1)}<\frac{n(n-1)-2 m+(n-1) \sqrt{n k-k^{2}}}{\sqrt{n-k}+\sqrt{k}}
$$

whose direct consequence is $K f(\bar{G})<L E L(\bar{G})$.

The importance of the Theorem 2.23 is that it is directly applicable to the complement $\bar{G}$ of the graph $G$ and, under the conditions stated in the theorem, is an improvement of the Theorem 2.12, for all $n \geq 9$. The condition $\mu_{1}(G) \leq \frac{n}{2}$, in Theorem 2.23 , by Lemma 2.3 , gives $\mu_{n-1}(\bar{G}) \geq \frac{n}{2}$. That is, this theorem is applicable to the graphs whose complements have a higher value of algebraic connectivity. As an instance, we have the following corollary.

Corollary 2.24. Let $T$ be a tree on $n \geq 41$ vertices with largest Laplacian eigenvalue $\mu_{1} \leq \frac{n}{2}$ and algebraic connectivity $\mu_{n-1} \geq 0.1$. Then $\operatorname{LE} L(\bar{T})>K f(\bar{T})$. 
Proof. For all $\mu_{1} \leq \frac{n}{2}$ and $n \geq 41$, by Theorem 2.23, we have

$$
2(n-1)<\frac{(\Delta+1)(n-\Delta-1)(n-1)(n+\sqrt{0.1(n-0.1)})}{n(\sqrt{n-0.1})+\sqrt{0.1}+(\Delta+1)(n-\Delta-1)} .
$$

which is clearly true, as for a tree $T$ of order $n$ has $n-1$ edges and $\Delta+1 \leq \mu_{1} \leq \frac{n}{2}$ gives $\Delta+1 \leq \frac{n}{2}$ and $n-\Delta-1 \geq \frac{n}{2}$.

We have partially solved the problem in [9] to find a constant $c$ (which may depend on the number of vertices $n$ and maximum vertex degree $\Delta$ ), such that for any connected graph $G$ with $m \geq c$ edges, $L E L(G)>K f(G)$. It will be of interest in future to find more sufficient conditions for the inequality $L E L(G)>K f(G)$.

Acknowledgement. The authors would like to thank the anonymous referee for his/her helpful suggestions.

\section{REFERENCES}

[1] W.N. Anderson and T.D. Morley. Eigenvalues of the Laplacian of a graph. Linear Multilinear Algebra, 18:141-145, 1985.

[2] C. Arauz. The Kirchhoff indexes of some composite networks. Discrete Appl. Math., 160:14291440, 2012.

[3] B. Arsić, I. Gutman, K.C. Das, and K. Xu. Relations between Kirchhoff index and Laplacianenergy-like invariant. Bull. Cl. Sci. Math. Nat. Sci. Math., 37:59-70, 2012.

[4] E. Bendito, A. Carmona, A.M. Encinas, J.M. Gesto, and M. Mitjana. Kirchhoff indexes of a network. Linear Algebra Appl., 432:2278-2292, 2010.

[5] D. Bonchev, A.T. Balaban, X. Liu, and D.J. Klein. Molecular cyclicity and centricity of polycyclic graphs. I. Cyclicity based on resistance distances or reciprocal distances. Internat. J. Quantum Chem., 50:1-20, 1994.

[6] D. Cvetkovic, M. Doob, and H. Sachs. Spectra of Graphs - Theory and Application. Academic Press, Inc., New York, 1980.

[7] K.C. Das, A.D. Güngör, and A.S. Çevik. On Kirchhoff index and the resistance-distance energy of a graph. MATCH Commun. Math. Comput. Chem., 67:541-556, 2012.

[8] K.C. Das, I. Gutman, and A.S. Çevik. On the Laplacian-energy-like invariant. Linear Algebra Appl., 442:58-68, 2014.

[9] K.C. Das, K. Xu, and I. Gutman. Comparison between Kirchhoff index and the Laplacianenergy-like invariant. Linear Algebra Appl., 436:3661-3671, 2012.

[10] N.M.M. de Abreu. Old and new results on algebraic connectivity of graphs. Linear Algebra Appl., 423:53-73, 2007.

[11] E. Estrada and N. Hatano. Topological atomic displacements, Kirchhoff and Wiener indices of molecules. Chem. Phys. Lett., 486:166-170, 2010.

[12] M. Fiedler. Algebraic connectivity of graphs. Czechoslovak Math. J., 23:298-305, 1973.

[13] M. Freitas, R. Del-Vecchio, and N. Abreu. Spectral properties of $K K_{n}^{j}$ graphs. Mat. Contemp., 39:129-134, 2010.

[14] X. Gao, Y. Luo, and W. Liu. Resistance distances and the Kirchhoff index in Cayley graphs. Discrete Appl. Math., 159:2050-2057, 2011.

[15] X. Gao, Y. Luo, and W. Liu. Kirchhoff index in line, subdivision and total graphs of a regular graph. Discrete Appl. Math., 160:560-565, 2012. 
[16] H.A. Ganie, S. Pirzada, and I. Antal. Energy and Laplacian energy of double graphs and new families of equienergetic graphs. Acta Univ. Sap. Informatica, 6:89-116, 2014.

[17] C. Godsil and G. Royle. Algebraic Graph Theory. Springer-Verlag, New York, 2001.

[18] R. Grone and R. Merris. The Laplacian spectrum of a graph II. SIAM J. Discrete Math., $7: 221-229,1994$

[19] R. Grone, R. Merris, and V.S. Sunder. The Laplacian spectrum of a graph. SIAM J. Matrix Anal. Appl., 11:218-238, 1990.

[20] I. Gutman. Estimating the Laplacian-energy-like molecular structure descriptor. Z. Naturforsch., 67a:403-406, 2012.

[21] I. Gutman and B. Mohar. The quasi-Wiener and the Kirchhoff indices coincide. J. Chem. Inf. Comput. Sci., 36:982-985, 1996.

[22] I. Gutman and B. Zhou. Laplacian energy of a graph. Linear Algebra Appl., 414:29-37, 2006.

[23] D.J. Klein and M. Randić. Resistance distance. J. Math. Chem., 12:81-95, 1993.

[24] R. Li. Lower bounds for the Kirchhoff index. MATCH Commun. Math. Comput. Chem., 70:163-174, 2013.

[25] X. Li, Y. Shi, and I. Gutman. Graph Energy. Springer, New York, 2012.

[26] B. Liu, Y. Huang, and Z. You. A survey on the Laplacian-energy-like invariant. MATCH Commun. Math. Comput. Chem., 66:713-730, 2011.

[27] J. Liu and B. Liu. A Laplacian-energy-like invariant of a graph. MATCH Commun. Math. Comput. Chem., 59:355-372, 2008.

[28] M. Liu, B. Liu, and X. Tan. The first to ninth greatest LEL-invariants of connected graphs. Util. Math., 93:153-160, 2014.

[29] R. Merris. Laplacian matrices of graphs: A survey. Linear Algebra Appl., 197/198:143-176, 1994.

[30] R. Merris. A survey of graph Laplacians. Linear Multilinear Algebra, 39:19-31, 1995.

[31] J.L. Palacios and J.M. Renom. Broder and Karlin's formula for hitting times and the Kirchhoff index. Int. J. Quantum Chem., 111:35-39, 2011.

[32] S. Pirzada. An Introduction to Graph Theory. Universities Press, Orient Blackswan, Hyderabad, 2012.

[33] S. Pirzada and H.A. Ganie. On the construction of L-equienergetic graphs. AKCE Int. J. Graphs Comb., 12,2:141-154, 2015.

[34] S. Pirzada and H.A. Ganie. On the Laplacian eigenvalues of a graph and Laplacian energy. Linear Algebra Appl., 486:454-468, 2015.

[35] S. Pirzada and H.A. Ganie. On Laplacian-energy-like invariant and incidence energy. Trans. Comb., 4:41-51, 2015.

[36] S. Pirzada and H.A. Ganie. Spectra, energy and Laplacian energy of strong double graphs. In: D. Mugnolo (editor), Mathematical Technology of Networks, Springer Proc. Math. Stat., Springer International Publishing, 128:175-189, 2015.

[37] S. Pirzada, H.A. Ganie, and E.T. Baskoro. On energy, Laplacian energy and $p$-fold graphs. Electronic J. Graph Theory Appl., 3:94-107, 2015.

[38] S. Pirzada, H.A. Ganie, and I. Gutman. On Laplacian-energy-like invariant and Kirchhoff index. MATCH Commun. Math. Comput. Chem., 73:41-59, 2015.

[39] W. Wang and Y. Luo. On Laplacian-energy-like invariant of a graph. Linear Algebra Appl., 437:713-721, 2012.

[40] W. Wang, D. Yang, and Y. Luo. The Laplacian polynomial and Kirchhoff index of graphs derived from regular graphs. Discrete Appl. Math., 161:3063-3071, 2013.

[41] K. Xu and K.C. Das. Extremal Laplacian-energy-like invariant of graphs with given matching number. Electron. J. Linear Algebra, 26:131-140, 2013.

[42] Y. Yang. The Kirchhoff index of subdivisions of graphs. Discrete Appl. Math., 171:153-157, 2014. 
Electronic Journal of Linear Algebra ISSN 1081-3810

A publication of the International Linear Algebra Society

Volume 31, pp. 27-41, January 2016

[43] Y. Yang and D.J. Klein. Comparison theorems on resistance distances and Kirchhoff indices of S, T-isomers. Discrete Appl. Math., 175:87-93, 2014.

[44] Z. You and B. Liu. On the Laplacian-energy-like of graphs. Ars Combin., 110:355-369, 2013.

[45] B.X. Zhu. The Laplacian-energy like of graphs. Appl. Math. Lett., 24:1604-1607, 2011. 